GENERALIZATIONS OF COMPLEX ANALYSIS

BANACH CENTER PUBLICATIONS, VOLUME 37

INSTITUTE OF MATHEMATICS

POLISH ACADEMY OF SCIENCES

WARSZAWA 1996

\title{
THE CLIFFORD BUNDLE AND THE DYNAMICS OF THE SUPERPARTICLE
}

\author{
WALDYR A. RODRIGUES, JR. \\ Department of Applied Mathematics \\ State University at Campinas (UNICAMP) \\ CP 6065, 13081-970 Campinas, S.P., Brazil \\ E-mail: walrod@ime.unicamp.br \\ JAYME VAZ, JR. \\ Department of Applied Mathematics \\ State University at Campinas (UNICAMP) \\ CP 6065, 13081-970 Campinas, S.P., Brazil \\ E-mail:vaz@ime.unicamp.br \\ MATEJ PAVSIC \\ J. Stefan Institute, University of Ljubljana \\ 61111 Ljubljana, Slovenia \\ E-mail: Matej.Pavsic@IJS.si
}

\begin{abstract}
Using the Clifford bundle formalism we show that Frenet equations of classical differential geometry or its spinor version are the appropriate equations of motion for a classical spinning particle. We show that particular values of the curvatures appearing in Darboux bivector of the spinor form of Frenet equations produce a "classical" Dirac-Hestenes equation. Using the concept of multivector Lagrangians and Hamiltonians we provide a Lagrangian and Hamiltonian approach for our theory which then makes immediately contact with Berezin-Marinov model, the Barut-Zanghi model, and the supercalculus (which acquires an obvious geometrical meaning in terms of geometrical objects living in ordinary spacetime) and suggests calling our theory the dynamics of the superparticle.
\end{abstract}

1. Introduction. Producing a classical spinning model that after quantization gives Dirac equation has always been a very appealing idea which has been the subject of several interesting papers containing new physical insights and beautiful mathematics.

1991 Mathematics Subject Classification: Primary 81R25; Secondary 53A50, 32L25.

The paper is in final form and no version of it will be published elsewhere. 
The most popular of such models is due to Berezin-Marinov [3], where they construct actions both for the non relativistic and relativistic spin dynamics supposing that the dynamical variables describing spin are elements of a Grassmann algebra. The paper uses the now famous supercalculus which starts with Berezin [2] and has been further developed in details with applications to supersymmetry [4,11] and superstring theory. Good references are $[4,6,11,41]$.

Another approach that has been the subject of many investigations is the BarutZanghi model (BZM) [1] which uses Dirac spinors in the classical action.

In the present paper using the Clifford bundle formalism (CBF) $[27,31,36]$ we show that Frenet equations (of classical differential geometry [14]), for a Frenet frame $\left\{f_{\mu}\right\}^{1}$ of a time-like curve in spacetime provide appropriate equations of motion for a classical spinning particle.

Frenet equations have already been used for the description of the relativistic kinematics of a classical point particle in a very beautiful paper by Gursey [13] in 1957. What is new in our approach, besides the use of the Clifford bundle formalism, is that using the concept of multivector Lagrangians used originally in $[8,12,25,26]$ and developed, e.g., in $[7,30]$, we succeded in formulating a Lagrangian and Hamiltonian theory for Frenet equations and thus for the classical spinning particle dynamics.

Our approach makes immediate contact with the Berezin-Marinov as well as the Barut-Zanghi model, ${ }^{2}$ clearing their physical as well as their mathematical contents. In particular the geometrical meaning (i.e., meaning in terms of geometrical objects living in ordinary spacetime) of the Grassmann variables and the supercalculus is disclosed, by showing the isomorphism of our multivector Lagrangian and the Berezin-Marinov Lagrangian for the 4-dimensional spacetime case.

Another important result showed below is the following: given a fiducial orthonormal frame for the spacetime $\left\{\gamma_{\mu}\right\}, \gamma_{\mu} \in \sec T M \subset \sec \mathcal{C} \ell(M, g)$, the Frenet frame $\left\{f_{\mu}\right\}$ is related to $\left\{\gamma_{\mu}\right\}$ by

$$
\rho f_{\mu}=\psi \gamma_{\mu} \tilde{\psi}
$$

where $\gamma_{\mu}$ is the restriction of $\gamma_{\mu}$ on $\sigma: \mathbb{R} \supset I \rightarrow M$ (see footnote 1 ), and $\psi$ is an inhomogeneous multivector field over the map $\sigma$, which is the restriction over $\sigma$ of $\boldsymbol{\psi} \in$ $\left.\sec \left(\wedge^{0}(T M)+\wedge^{2}(T M)+\wedge^{4}(T M)\right)\right) \subset \sec \mathcal{C} \ell(M, g) ; \boldsymbol{\psi}$ is called the representative of the Dirac-Hestenes spinor field [31] in the fiducial basis $\left\{\gamma_{\mu}\right\}$. (In what follows, for short, we refer to $\boldsymbol{\psi}$ simply as a DHS). $\boldsymbol{\psi}$ has a canonical decomposition [14,31] when $\boldsymbol{\psi} \tilde{\boldsymbol{\psi}} \neq 0$ as

$$
\boldsymbol{\psi}=\sqrt{\rho} e^{\beta \gamma_{5} / 2} U
$$

where $\rho(x) \in \mathbb{R}_{+}, 0 \leq \beta(x)<2 \pi$ and $U(x) \subset \operatorname{Spin}_{+}(1,3) \subset \mathcal{C} \ell_{1,3}^{+}, \forall x \in M, \gamma_{5}=$ $\gamma_{0} \gamma_{1} \gamma_{2} \gamma_{3} \cdot \mathcal{C} \ell_{1,3}$ is the spacetime algebra $[9,10,18]$ and $\mathcal{C} \ell_{1,3}^{+} \simeq \mathcal{C} \ell_{3,0}$ is the even subalgebra

\footnotetext{
${ }^{1} f_{\mu},(\mu=0,1,2,3)$ are a set of vector fields over a map [36] $\sigma: \mathbb{R} \supset I \rightarrow M$ (where $\sigma$ is a time-like curve in the spacetime $(M D, g))$, which are section of $\mathcal{C} \ell(M, g)$, the Clifford bundle of multivections $[27,36]$ over $(M, D, g)$.

2 BZ model has already been atudied by the authors in $[28,29]$ using CBF.
} 
of $\mathcal{C} \ell_{1,3}$ isomorphic to the Pauli algebra. $\mathrm{Eq}(2)$ discloses the hidden geometrical meaning of Dirac spinors.

Now, Frenet equations for the $\left\{f_{\mu}\right\}$ are

$$
\frac{D}{d \tau} f_{\mu}=\Omega \cdot f_{\mu}, \quad(\tau \subset I)
$$

where $\Omega$ is the so called Darboux bivector [14], given by

$$
\Omega=K_{0} f^{1} \wedge f^{0}+K_{1} f^{2} \wedge f^{1}+K_{2} f^{3} \wedge f^{2}
$$

where $K_{i},(i=0,1,2)$ is the $i$-curvature, which is the projection of $\Omega$ in the $f_{i} \wedge f_{i-1}$ plane, and · in eq. (3) is the dot product (or contraction [18]) in the Clifford algebra.

We show that the validity of Frenet equations for a congruence of worldlines and for some particular values of the curvatures appearing in eq. (4) leads to the so called Dirac-Hestenes equation for $\boldsymbol{\psi}$ (related to the $f_{\mu}$ by eq. (2)), which as is now well known is the representative in the Clifford bundle of the usual Dirac equation.

2. Mathematical background. Let $(M, D, g)$ be a relativistic spacetime $[22,34], D$ being the Levi-Civita connection of $g . \forall x \in M, T_{x} M=\mathbb{R}^{1,3}$, where $\mathbb{R}^{1,3}$ is the Minkowski vector space [22]. $\mathcal{C} \ell(M, g)$ is the Clifford bundle over the relativistic spacetime $(M, D, g)$. Details on the structure of $\mathcal{C} \ell(M, g)$ can be found e.g., in $[9,10,36]$ and in what follows we use the notations and conventions of $[9]^{3}$. Sections of $\mathcal{C} \ell(M, g)$ are called multivector fields. These objects are isomorphic to the superfields introduced by Salam and Strathdee [35] (see also [41]) as is clear from their definition and the isomorphisms we exhibit in Section 6. (It is amazing to discover that $\boldsymbol{\psi}$, the Dirac-Hestenes spinor is a superfield!). Then, if $\left\{\gamma_{\mu}\right\}, \gamma_{\mu} \in \sec \wedge^{1}(T M) \subset \sec \mathcal{C} \ell(M, g)$ is an orthonormal basis for $M$, and if $X \in \sec \mathcal{C} \ell(M)$, we have

$$
X=X_{0}+X_{1}+X_{2}+X_{3}+X_{4}, X_{i} \in \sec \wedge^{i}(T M) \subset \sec \mathcal{C} \ell(M, g),
$$

or

$$
X=X_{0}+\left(X_{1}\right)^{\mu} \gamma_{\mu}+\frac{1}{2}\left(X_{2}\right)^{\mu \nu} \gamma_{\mu} \wedge \gamma_{\nu}+\frac{1}{3 !}\left(X_{3}\right)^{\mu \nu \rho} \gamma_{\mu} \wedge \gamma_{\nu} \wedge \gamma_{\rho}+S \gamma_{0} \gamma_{1} \gamma_{2} \gamma_{3}
$$

In what follows all fields used are sections of $\mathcal{C} \ell(M, g)$ or are Clifford fields over the map $\sigma: \mathbb{R} \subset I \rightarrow M$, where $\sigma$ is a time-like vector curve pointing into the future. We need the following definitions which have been adapted from definitions appearing originally in $[34]$.

Definition 1. An observer in $(M, D, g)$ is a time-like curve $\sigma: \mathbb{R} \supset I \rightarrow M$ pointing into the future, such that the assignment $\tau \mapsto \sigma_{*} \tau \in I$ is by definition the tangent vector field of $\sigma$, denoted simply by $\sigma_{*}$ and $g\left(\sigma_{*}, \sigma_{*}\right)=1.4$

Definition 2. An instantaneous observer is a pair $(z, Z), z \in M$ and $Z \in T_{z} M$ is a time like vector field pointing to the future.

\footnotetext{
3 The only difference is that $\mathcal{C} \ell(M, g)$ refers to the Clifford bundle of differential forms in [9] and here it is the Clifford bundle of multivectors.

${ }^{4} \sigma_{*}$ is an example of a tensor field under a map $\sigma$.
} 
Definition 3. A reference system in $(M, D, g)$ is a time like vector field defined in an open set $U \subseteq M$ such that each of its integral lines is an observer.

Then, if $Q \in \sec T U \subset \sec T M \subset \sec \mathcal{C} \ell(M, g)$ is a reference frame, then $g(Q, Q)=$ $Q \cdot Q=1$ where the symbol " . " is the dot product in the Clifford bundle $[9,10]$.

Definition 4. A moving system for $x \in M$ is an orthonormal basis for $T_{x} M$. A moving system for all points $x \in \sigma$ (of definition 1) will be called a comoving frame for $\sigma$.

If $\left\{e_{\mu}\right\}, \mu=0,1,2,3$ is a comoving frame for $\sigma$, such that $e_{0}=\sigma_{*}$, then it can be proved that there exists a unique bivector field $\Omega$ over $\sigma$, called the angular velocity, such that the $e_{\mu}$ satisfies the following system of differential equations

$$
\begin{aligned}
\frac{D}{d \tau} e_{\mu} & =\Omega \cdot e_{\mu}, \\
\Omega=\frac{1}{2} \omega_{\mu \nu} e_{\mu} \wedge e_{\nu} & =\frac{1}{2} \dot{e}_{\mu} e^{\mu}=\frac{1}{2} \dot{e}_{\mu} \wedge e_{\mu} .
\end{aligned}
$$

For reasons that will be come clear in what follows we call eq. (6) the superparticle equations of motion.

Definition 5. Let $(z, Z)$ be an instantaneous observer. Span $Z$ is his local time axis and $Z^{\perp}$ is called his local rest frame and the direct sum $T_{z} M=\operatorname{Span} Z \oplus Z^{\perp}$ is the local decomposition of $T_{z} M$ in time and space.

If $p: T_{z} M \rightarrow Z^{\perp}$ denotes the orthogonal projection and $\sigma: \mathbb{R} \subset I \rightarrow M$ is an observer then corresponding terms for the instantaneous observer $\left(\sigma \tau, \sigma_{*} \tau\right)$ are used.

DeFinition 6. The acceleration of $\sigma$ is given by

$$
a_{\sigma} \tau=D_{\sigma_{*}} \sigma_{*} \tau
$$

or, simply, $a=D_{\sigma_{*}} \sigma_{*}$.

DEFINITION 7. The projection tensor $h$ for an instantaneous observer is such that $h(X, Y)=g(p X, p Y) \quad \forall X, Y \in T_{Z} M$, where $p$ is projection tensor $p: T_{z} M \rightarrow Z^{\perp}$.

As it is well known for an observer $\sigma$ to decide when a unitary vector $X \in\left(\sigma_{*} \tau\right)^{\perp}$ has the same spatial direction of the unitary vector $X^{\prime} \in\left(\sigma_{*} \tau^{\prime}\right)^{\perp}\left(\tau^{\prime} \neq \tau\right)$, he has to introduce the concept of the Fermi-Walker connection. We have,

Definition 8. Let $\sigma: \mathbb{R} \supset I \rightarrow M$ be an observer and write $T_{\sigma u} M=T_{u} \oplus R_{u}, u \in I$. Define $p_{u}=T_{\sigma u} M \rightarrow R_{u}, q_{u}: T_{\sigma u} M \rightarrow T_{u}$. If $X$ is a vector field over $\sigma$ then $p X$ and $q X$ are vector fields over $\sigma$ given by $(p X)_{u}=p_{u}\left(X_{u}\right),(q X)_{u}=q_{u}\left(X_{u}\right)$.

Proposition [34]. There exists one and only one connection over $\sigma$, such that

$$
F_{y} X=\left[p\left(\sigma^{*} D\right)_{y} p+q\left(\sigma^{*} D\right)_{y} q\right] X,
$$

for all vector fields $Y$ in $I(\sigma: \mathbb{R} \supset I \rightarrow M)$ and for all vector fields over $\sigma$.

Also $\sigma^{*} D$ is the pullback of the Levi-Civita connection $D$ and $F$ is called the FermiWalker connection over $\sigma$, and we shall write $F_{\sigma_{*}}, F / d \tau$ or $F_{e_{o}}, e_{o}=\sigma_{*}$ when convenient. We also shall write $D$, as usual, for $\sigma^{*} D$ in what follows. 
Proposition [34]. Let be $X, Y$ are vector fields over $\sigma$, interpreted as sections of the Clifford bundle. The Fermi-Walker connection $F$ satisfies the properties

(a) $\quad F_{e_{o}} X=D_{e_{0}} X-\left(e_{0} \cdot X\right) a+(a \cdot X) e_{0}$,

where $a=D_{e_{0}} e_{0}$, is the acceleration.

(b) $\frac{d}{d \tau} X \cdot Y=F_{e_{0}} X \cdot Y+X \cdot F_{e_{0}} Y$.

(c) $\quad \stackrel{d}{F}_{e_{o}} e_{0}=0$.

(d) If $X_{u} \in R_{u} \forall u \in I$, then $F_{e_{0}} X \in R_{u}, \forall u$, and

$$
F_{e_{0}} X \cdot Y=D_{e_{0}} X \cdot Y \text {. }
$$

As is well known if $X$ is a space-like vector field over $\sigma$ it will be transported without rotation over $\sigma$ if and only if $F_{e_{0}} X=0$. If this is the case then

$$
D_{e_{0}} X \equiv \frac{D}{d \tau} X=\left(e_{0} \cdot X\right) a+(a \cdot X) e_{0}=\left(a \wedge e_{0}\right) \cdot X
$$

3. Frenet equations and their physical meaning. We already defined a comoving frame over $\sigma$ (Definition 4). We now define a Frenet frame over $\sigma$.

Definition 9. A comoving frame $\left\{f_{\mu}\right\}$ over $\sigma$ is called a Frenet frame over $\sigma$ if and only if the $f_{\mu}$ satisfy the Frenet equations:

$$
D_{f_{0}} f_{\mu}=\Omega \cdot f_{\mu}
$$

with $\Omega$ given by ${ }^{5}$

$$
\Omega=K_{0} f^{1} \wedge f^{0}+K_{1} f^{2} \wedge f^{1}+K_{2} f^{3} \wedge f^{2},
$$

is the Darboux bivector. $K_{i}$ is called the $i-$ th curvature and is the projection of $\Omega$ on the $f^{i} \wedge f^{i-1}$ plane.

It is quite clear that after eq. (6), eq. (7) and eq. (8) are particular superparticle equations of motion.

From eq. (7) and eq. (8) it is clear that $a=D_{f_{0}} f_{0}=K_{0} f^{1}$.

Then we can write $\Omega$ as

$$
\Omega=a \wedge f_{0}+\Omega_{R}=\Omega_{F}+\Omega_{R},
$$

where $\Omega_{R}$ is a bivector which can be directly associated with the intrinsic spin bivector of a classical spinning particle as shown in what follows. Indeed, suppose that $\left\{b_{\mu}\right\}$ is a comoving frame over $\sigma$ such that $F_{b_{0}} b_{\mu}=0$.

Then

$$
D_{b_{0}} b_{\mu}=\left(a \wedge b_{0}\right) \cdot b_{\mu},
$$

and comparing eq. (9) with Frenet equations eq. (7) it follows that in this case $\Omega=\Omega_{F}$.

It is then clear that since $b_{\mu}, i=1,2,3$ can be interpreted as gyroscopic axis [34] that a general Frenet frame $\left\{f_{\mu}\right\}$ over $\sigma$ rotates with bivector angular velocity $\Omega_{R}$ relative to the tetrad $\left\{b_{\mu}\right\}$ which is Fermi-Walker transported.

\footnotetext{
${ }^{5}$ Observe that since the $f_{\mu}$ are orthonormal we can write $\Omega=K_{0} f^{1} f^{0}+K_{1} f^{2} f^{1}+K_{3} f^{3} f^{2}$, i.e., we can substitute the $\wedge$ product by the Clifford product denoted by justaposition $[9,10]$.
} 
It is then obvious that $\Omega_{R}$ is (a dimensional appart) the spin bivector being naturally associated to the so called Spin vector $[20,40]$ (also called the Pauli-Lubanski-Bargmann vector [5]). To show this, let us observe that $\Omega_{R} \cdot f_{0}=\Omega \cdot \sigma_{*}=\Omega \cdot v=0$. Indeed,

$$
\Omega_{R} \cdot v=\left(K_{1} f^{2} \wedge f^{1}+K_{2} f^{3} \wedge f^{2}\right) \cdot f_{0}=0 .
$$

Now, let $\star \Omega_{R}$ be the Hodge dual of $\Omega_{R}$. In the Clifford bundle formalism $[27,36]$ we can write $\left(f_{5}=f_{0} f_{1} f_{2} f_{3}\right)$

$$
\star \Omega_{R}=-\Omega_{R} f_{5} .
$$

Defining the rotation vector by

$$
R=-\star \Omega_{R} \cdot v=v \cdot\left(\star \Omega_{R}\right),
$$

we immediately get that

$$
R \cdot v=0 .
$$

Indeed, using the identity [14] $A_{r} \cdot\left(B_{s} \cdot C_{t}\right)=\left(A_{r} \wedge B_{s}\right) \cdot C_{t}, r+s \leq t, r, s>0$ where $A_{r} \in \sec \wedge^{r}(T M) \subset \sec \mathcal{C} \ell(M, g)$, etc..., we have

$$
\left(\star \Omega_{R} \cdot v\right) \cdot v=v \cdot\left(\star \Omega_{R} \cdot v\right)=-v \cdot\left(v \cdot \star \Omega_{R}\right)=-(v \wedge v) \cdot \star \Omega_{R}=0 .
$$

Since $R \cdot v=0$, then in a reference frame comoving with the particle, $v$ has only spatial components and it follows that $R$ is a spacelike vector.

From eq. (10) we have that

$$
D_{f_{0}}\left(R \cdot f_{0}\right)=0,
$$

from where it follows that $\left(D_{f_{0}} R\right) \cdot f_{0}=-R \cdot a$ and then

$$
D_{e_{0}} R=-R \cdot\left(a \wedge f_{0}\right) \Leftrightarrow F_{f_{0}} R=0 .
$$

From these results it is clear that the spin-vector $\boldsymbol{S}$ (the Pauli-Lubanski-Bargmann vector) of the classical spinning particle is given by

$$
\boldsymbol{S}=k \hbar R \quad(\hbar=1),
$$

where $k$ is a real number, $\hbar$ is the Planck constant, inserted here for dimensional reason. As is well known [20,40] $D_{f_{0}} \boldsymbol{S}=-\boldsymbol{S} \cdot\left(a \wedge f_{0}\right)$ is the equation of motion of the intrinsic spin of a classical spinning particle being accelerated $\left(a=D_{f_{0}} f_{0}\right)$ by a force producing no torque.

4. The spinor form of Frenet equations. In this section let $(M, D, g)$ be Minkowski spacetime and $\gamma_{\mu}=\partial / \partial x^{\mu}$ a global orthonormal basis for $T M$, i.e, $\gamma_{\mu} \in \sec \wedge^{1}(T M)$ $\subset \sec \mathcal{C} \ell(M, g)$.

Recalling eq. (1) and eq. (2) we see that the Frenet frame over $\sigma$ can be expressed as $f_{\mu}=\psi \gamma_{\mu} \tilde{\psi}$, where $\psi$ is a unitary Dirac-Hestenes spinor field over $\sigma$, that is, $\psi \tilde{\psi}=1$, $\psi(\tau) \in \operatorname{Spin}_{+}(1,3), \forall \tau \in \mathbb{R}$.

From Frenet equations that now reads $\dot{f}_{\mu}=\Omega \cdot f_{\mu}$, we immediately obtain the equation of motion for $\psi$ as

$$
\frac{d}{d \tau} \psi=\frac{1}{2} \Omega \psi
$$


This is the spinor form of the Frenet equations. It is obvious that an analogous equation can be deduced also from eq. (6), the general superparticle equations of motion.

5. "Classical" Dirac-Hestenes equation. Consider now a free classical spinning particle. Then $\dot{f}_{0}=0$ and we can take without any loss of generality $f_{0}=\gamma_{0}$ where $\gamma_{0}=\gamma_{0 \mid \sigma}$. In this case the Darboux bivector is simply

$$
\Omega=K_{1} f^{2} \wedge f^{1}+K_{2} f^{3} \wedge f^{2}
$$

and let us study the spinor equation of motion (eq. (12)) under the condition $K_{2}=0 .{ }^{6}$

Then eq. (12) gives

$$
\frac{d \psi}{d \tau}=\frac{1}{2} K_{1} f_{2} f_{1} \psi=\frac{1}{2} K_{1} \psi \gamma_{2} \gamma_{1}
$$

which has a solution ${ }^{7}$

$$
\psi=\exp \left(\frac{K_{1}}{2} \gamma_{2} \gamma_{1} \tau\right) .
$$

Now, let us suppose that there exists $\psi \in \sec \left(\wedge^{0}(T M)+\wedge^{2}(T M)+\wedge^{4}(T M)\right) \subset$ $\sec \mathcal{C} \ell(M, g)$ such that $\psi=\boldsymbol{\psi}_{\mid \sigma}$. Being $\partial=\gamma^{\mu} \nabla_{\gamma_{\mu}}$ the Dirac operator acting on sections of the Clifford bundle and writting $x=x^{\mu} \gamma_{\mu},\left\langle x^{\mu}\right\rangle$ being the global Lorentz chart introduced above and defining $p=K_{1} e_{0}$, eq. (14) can be written (since $d / d \tau=f_{0} \cdot \partial=\gamma_{0} \cdot \partial$ ) as

$$
f_{0} \cdot \partial \boldsymbol{\psi}=\frac{K_{1}}{2} \boldsymbol{\psi} \gamma_{2} \gamma_{1}
$$

or

$$
\gamma_{0} \cdot \partial \boldsymbol{\psi}=\frac{K_{1}}{2} \boldsymbol{\psi} \gamma_{2} \gamma_{1}
$$

and

$$
\boldsymbol{\psi}=e^{\gamma_{2} \gamma_{1} p x}
$$

is a solution. Also

$$
\partial_{\mu} \boldsymbol{\psi}=p_{\mu} \boldsymbol{\psi} \gamma_{2} \gamma_{1}
$$

Then,

$$
f_{0} \cdot \partial \boldsymbol{\psi}=\gamma_{0} \cdot \partial \boldsymbol{\psi}=\gamma_{0} \partial \boldsymbol{\psi}=\left(\boldsymbol{\psi} \gamma_{0} \boldsymbol{\psi}^{-1}\right) \partial \boldsymbol{\psi}
$$

Using eq. (16) in eq. (15) gives

$$
\partial \boldsymbol{\psi} \gamma_{2} \gamma_{1}+\frac{K_{1}}{2} \boldsymbol{\psi} \gamma_{0}=0
$$

If we identify with appropriate units $\frac{K_{1}}{2}=\frac{m}{\hbar}$, the mass of the spinning particle, we get

$$
\hbar \partial \boldsymbol{\psi} \gamma_{2} \gamma_{1}+m \boldsymbol{\psi} \gamma_{0}=0
$$

${ }^{6}$ It is important to observe that this is a very reasonable assumption; indeed, it has been shown in [38] that all worldlines in Barut and Zanghi model (sec.6.2) have vanishing third curvature $\left(K_{2}=0\right)$.

7 This equation has other solutions, see [21], and comments at the end of the section. 
which is the Dirac-Hestenes equation for the unitary spinor $\boldsymbol{\psi}$. If $S$ is the spin bivector associated to the spin vector defined by eq. (11) and $\star S$ its Hodge dual, i.e.,

$$
\boldsymbol{S}=\star S \cdot v=\star S \cdot e_{0}=\star S \cdot \gamma_{0},
$$

then

$$
S=-k \hbar \psi \gamma_{2} \gamma_{1} \tilde{\psi}
$$

and since $m=p \cdot v=p \cdot f_{0}$ we get using eq. (13) and eq. (17):

$$
\Omega \cdot S=2 k m
$$

It follows that if $k=1 / 2$ we can write $p \cdot v=\Omega \cdot S=m$, an equation that suggests that the mass of a $1 / 2$ spinning particle has "internal" kinetic origin $[15,28,29]$. It is interesting to note that this value for $k=1 / 2$ implies the natural unity of angular momentum $\hbar / 2$ for the Pauli - Lubanski - Bargmann vetor $S=\frac{\hbar}{2} R$ (eq. (11)).

Before ending this section let us observe that the Dirac-Hestenes equation is satisfied by a general Dirac-Hestenes spinor field given by eq. (2), i.e., by

$$
\boldsymbol{\psi}=\sqrt{\rho} e^{\gamma_{5} \beta / 2} R
$$

where $\rho$ is the density function and $\beta$ is the Takabayasi angle [37] and $R(x) \in \operatorname{Spin}_{+}(1,3)$ $\forall x \in M$. It has been proved recently [39] that the transformations $e^{\gamma_{5} \beta / 2}$ are directly associated with the duality transformations of generalized electromagnetism thereby $[8,12,23,24]$ providing a Clifford algebra proof for the Rainich-Misner-Wheeler Theorem [39]. From that theorem we know that given $P \in \sec \wedge^{2}(T M) \subset \sec \mathcal{C} \ell(M, g)$ such that $P^{2} \neq 0$, then in each point $x \in M$ (Minkowski spacetime) $\Omega$ can be reduced to the form $P^{\prime}=\omega \gamma_{2} \gamma_{1}$, (where $\omega$ is a real function) by a local active Lorentz transformation plus a local duality transformation $e^{\alpha \gamma_{5}}$, i.e, we can write $P^{\prime}=e^{\gamma_{5} \beta / 2} \tilde{U} \Omega U e^{\gamma_{5} \beta / 2}$, where $U(x) \in \operatorname{Spin}_{+}(1,3) \simeq S \ell(2, \mathscr{C})$ is the Lorentz transformation.

The general superparticle equation of motion can be written as an equivalent equation in terms of a general Dirac-Hestenes spinor field in interaction with an electromagnetic field. This very interesting topic will be described in a following paper [32].

To end this section, we recall that eq. (14) is the equation of motion for a free spinning particle in the Barut-Zanghi model [1], which has solutions containing a zitterbewegung motion. The results found above prove without any doubt that contrary to what is thought the helicoidal motion which is a solution of the model is only partially responsible for the origin of the spin. Besides that there is also the intrinsic spin present even when particle trajectories are straight lines. This is a very important conclusion (see also [38]).

6. Multivector Lagrangian formalism. Up to now we have proved that Frenet equation and the Dirac-Hestenes equation are appropriate equations of motion for a classical spinning particle. We proceed by developing a Lagrangian and Hamiltonian formalism for that equations.

In order to do that we must introduce the concept of multivector Lagrangians. It involves the concept of multivector derivatives first introduced by Hestenes and Sobczyk [14] and briefly recalled in the appendix. 
In what follows we shall call the classical spinning particle, the superparticle for reasons that well be clear in a while. For generality in this section we consider a $n$ dimensional spacetime, i.e, $T_{x} M=\mathbb{R}^{1, n-1}$. We have,

Definition 10. A superparticle is a pair $(\sigma, X)$, where $\sigma: \mathbb{R} \supset I \rightarrow M$ is a time-like curve and $X: \mathbb{R} \supset I \rightarrow \sec \mathcal{C} \ell(M, g)$ is a Clifford-field over $\sigma$ (or a set of Clifford fields over $\sigma$ ).

DEFINITION 11. A multivector Lagrangian is a mapping

$$
L:(X(s), \dot{X}(s)) \mapsto L(X(s), \dot{X}(s)) \in \mathcal{C} \ell(M, g),
$$

where $X$ is as in eq. (5) and $s$ is an invariant time parameter on $\sigma$. $L$ is a multivector functional, i.e., it has values in the Clifford algebra $C l_{1, n-1}$ for each $s$.

The most general $L$ can then be written as

$$
L=\sum_{k}\langle L\rangle \equiv \sum_{k} L_{k}
$$

To gain confidence in the multivector derivative calculus we start by studying the most simple case, namely, we choose $X=X_{r} \in \sec \wedge^{r}(M) \subset \sec \mathcal{C} \ell(M, g)$ and $L=\langle L\rangle_{0}$, a scalar multivector functional.

We postulate next that the action for the superparticle is

$$
\mathcal{A}\left(X_{r}\right)=\int_{s_{1}}^{s_{2}} d s L\left(X_{r}(s), \dot{X}_{r}(s)\right),
$$

and that the equations of motion can be derived from the principle of stationary action, that reads (see Appendix for the concept of multivector derivative)

$$
\left.\frac{d}{d t} \mathcal{A}\left(X_{r}+t A_{r}\right)\right|_{t=0}=A_{r} * \partial_{X_{r}} S\left(X_{r}\right)=0,
$$

where $A_{r} \in \sec \wedge^{r}(T M) \subset \sec \mathcal{C} \ell(M, g)$ is a Clifford field over $\sigma$ such that $A_{r}\left(s_{1}\right)=$ $A_{r}\left(s_{2}\right)=0$. From eq. (18) we get

$$
\int_{s_{1}}^{s_{2}} d s\left[\left(A_{r} * \partial_{X_{r}}\right) L+\dot{A}_{r} * \partial_{\dot{X}_{r}} L\right]=0 .
$$

Since $L=\langle L\rangle_{0}$ and $X_{r}, A_{r} \in \sec \wedge^{r}(T M) \subset \sec \mathcal{C} \ell(M, g)$ then

$$
\begin{aligned}
& A_{r} * \partial_{X_{r}}\langle L\rangle_{0}=\left\langle A_{r}\left(\partial_{X_{r}} L\right)\right\rangle_{0}=A_{r} \cdot\left(A_{X_{r}} L\right)_{r}, \\
& \dot{A}_{r} * \partial_{\dot{X}_{r}}\langle L\rangle_{0}=\left\langle\dot{A}_{r}\left(\partial_{\dot{X}_{r}} L\right)\right\rangle_{0}=\dot{A}_{r} \cdot\left(A_{\dot{X}_{r}} L\right)_{r} .
\end{aligned}
$$

Using eq. (20) into eq. (19) results

$$
\int_{s_{1}}^{s_{2}} d s\left[\left(A_{r} \cdot\left(\partial_{X_{r}} L\right)_{r}-A_{r} \cdot \frac{d}{d s}\left(\partial_{\dot{X}_{r}} L\right)_{r}\right]=0,\right.
$$

i.e.,

$$
A_{r} \cdot\left\langle\partial_{X_{r}} L-\frac{d}{d s}\left(\partial_{\dot{X}_{r}} L\right)\right\rangle_{r}=0 .
$$


Since for $L=\langle L\rangle_{0}$, we have $\partial_{X_{r}} L=\left\langle\partial_{X_{r}} L\right\rangle_{r}$ and $\partial_{\dot{X}_{r}} L=\left\langle\partial_{\dot{X}_{r}} L\right\rangle$ and since $A_{r}$ is arbitrary then eq. (21) implies

or

$$
\left\langle\partial_{X_{r}} L-\frac{d}{d s}\left(\partial_{\dot{X}_{r}} L\right)\right\rangle_{r}=0
$$

$$
\partial_{X_{r}} L-\frac{d}{d s}\left(\partial_{\dot{X}_{r}} L\right)=0
$$

that is the Euler-Lagrange equation. It is quite clear that the eq. (22) holds for $L$ being a functional of a general Clifford field $X$ over $\sigma$.

Next we study the case where

$$
L=\sum_{k}\langle L\rangle_{k}=\sum_{k} L_{k},
$$

and we restrict ourselves without loss of generality to the case that $X=X_{r} \in \sec \wedge^{r}(T M)$ $\in \sec \mathcal{C} \ell(M, g)$.

We define

$$
\tau=\sum\langle\tau\rangle_{k}=\sum \tau_{k}
$$

where the $\tau_{k} \in \sec \wedge^{k}(M)$ are constant multivectors.

Then,

$$
\langle L \tau\rangle_{0}=L * \tau=\sum_{k} L_{k} * \tau_{k}=\sum_{k} L_{k} \cdot \tau_{k} .
$$

In this way $\langle L \tau\rangle_{0}$ has the role of a scalar valued Lagrangian and we define the action by

$$
\mathcal{A}(X)=\int_{s_{1}}^{s_{2}} d s\langle L(X, \dot{X}) \tau\rangle_{0} .
$$

Then, the principle of stationary action gives

$$
\begin{aligned}
& \int_{s_{1}}^{s_{2}} d s {\left[\left(A_{r} * \partial_{X}\right)\langle L \tau\rangle_{0}+\left(\dot{A}_{r} * \partial_{\dot{X}}\right)\langle L \tau\rangle_{0}\right] } \\
&=\sum_{k} \int_{s_{1}}^{s_{2}} d s\left[\left(A_{r} * \partial_{X}\right)\left(L_{k} * \tau_{k}\right)+c\left(\dot{A}_{r} * \partial_{\dot{X}}\right)\left(L_{k} * \tau_{k}\right)\right]=0 .
\end{aligned}
$$

Since $\left(A_{r} * \partial_{X}\right) \tau_{k}=0$, we have

$$
\begin{aligned}
\left(A_{r} * \partial_{X}\right)\left(L_{k} * \tau_{k}\right) & =\left[\left(A_{r} * \partial_{X}\right) L_{k}\right] * \tau_{k} \\
& =\left[\left(A_{r} * \partial_{X}\right) L_{k}\right]_{k} \cdot \tau_{k}=\left[A_{r}\left(\partial_{X} L_{k}\right)\right]_{k} \cdot \tau_{k} .
\end{aligned}
$$

But, since $X=X_{r} \in \sec \wedge^{r}(T M) \subset \sec \mathcal{C} \ell(M, g)$, then

$$
\partial_{X} L_{k}=\left(\partial_{X} L_{k}\right)_{|r-k|}+\left(\partial_{X} L_{k}\right)_{|r-k|+2}+\ldots+\left(\partial_{X} L_{k}\right)_{r+k},
$$

and we can write,

$$
\begin{gathered}
\left(A_{r} * \partial_{X}\right)\left(L_{k} * \tau_{k}\right)=\left\langle A_{r}\left(\partial_{X} L_{k}\right)_{\mid r-k}\right\rangle_{k} \cdot \tau_{k} \\
+\left\langle A_{r}\left(\partial_{X} L\right)_{|r-k|+2}\right\rangle_{k} \cdot \tau_{k}+\ldots+\left\langle A_{r}\left(\partial_{X} L_{k}\right)_{r+k}\right\rangle_{k} \cdot \tau_{k} \\
=\sum_{\ell=0}^{\frac{1}{2}(r+k-|r-k|)}\left\langle A_{r}\left(\partial_{X} L_{k}\right)_{|r-k|+2 \ell}\right\rangle \cdot \tau_{k} .
\end{gathered}
$$


Also,

$$
\begin{aligned}
& \left(\dot{A}_{r} * \partial_{\dot{X}}\right)\left(L_{k} * \tau_{k}\right)=\left[\dot{A}_{r}\left(\partial_{\dot{X}} L_{k}\right)\right]_{k} \cdot \tau_{k} \\
& =\sum_{\ell=0}^{\frac{1}{2}[r+k-|r-k|]}\left\langle\dot{A}_{r}\left(\partial_{\dot{X}} L_{k}\right)_{|r-k|+2 \ell}\right\rangle_{k} \cdot \tau_{k} \\
& =\sum_{\ell=0}^{\frac{1}{2}[r+k-|r-k|]}\left[\frac{d}{d s}\left\langle A_{r}\left(\partial_{\dot{X}} L_{k}\right)_{|r-k|+2 \ell}\right\rangle_{k}-\left\langle A_{r} \frac{d}{d s}\left(\partial_{\dot{X}} L_{k}\right)_{|r-k|+2 \ell}\right\rangle_{k}\right] \cdot \tau_{k} .
\end{aligned}
$$

Using eq. (24) and eq. (25) into eq. (23) and taking into account that $A_{r}\left(s_{1}\right)=$ $A_{r}\left(s_{2}\right)=0$ we get

$$
\sum_{k} \int_{s_{1}}^{s_{2}} d s \sum_{\ell=0}^{\frac{1}{2}[r+k-|r-k|]}\left\langle A_{r}\left[\left(\partial_{X} L_{k}\right)_{|r-k|+2 \ell} \frac{d}{d s}\left(\partial_{\dot{X}} L_{k}\right)_{|r-k|+2 \ell}\right\rangle_{k} \cdot \tau_{k}=0 .\right.
$$

Now, the $\tau_{k}$ are constant sections of $\wedge^{k}(M) \subset \mathcal{C} \ell(M)$. Then if $p=\left(\begin{array}{l}n \\ k\end{array}\right), \tau_{k}$ is of the form $\left(\tau_{k}\right)^{\mu_{1} \ldots \mu_{p}} \gamma_{\mu_{1}} \ldots \gamma_{\mu_{p}}$ where $\left(\tau_{k}\right)^{\mu_{1} \ldots \mu_{p}}$ are arbitrary real constants. Also the term in the brackets in eq. (26) is of the form \langle\rangle$_{k}=\left(\langle\rangle_{k}\right)^{\mu_{1} \ldots \mu_{p}} \gamma_{\mu_{1}} \ldots \gamma_{\mu_{p}}$ and eq. (26) results in a sum of terms of the form $(\tau)^{\mu_{1} \ldots \mu_{p}}\left(\langle\rangle_{k}\right) \mu_{1} \ldots \mu_{p}$. Since the $\left(\tau_{k}\right)^{\mu_{1} \ldots \mu_{p}}$ are arbitrary constants, eq. (26) implies that for each $k$ we must have

$$
\left\langle A_{r}\left[\partial_{X} L_{k}-\frac{d}{d s}\left(\partial_{\dot{X}} L_{k}\right)\right]_{|r-k|}+\ldots+\left[\partial_{X} L_{k}-\frac{d}{d s}\left(\partial_{X} L_{k}\right)-\frac{d}{d s}\left(\partial_{\dot{X}} L_{k}\right)\right]_{r+k}\right\rangle_{k}=0,
$$

or

$$
\left\langle A_{r}\left[\partial_{X} L_{k}-\frac{d}{d s}\left(\partial_{\dot{X}} L_{k}\right)\right]\right\rangle_{k}=0 .
$$

Now we show that eq. (27) implies the multivector Euler-Lagrange equation

$$
\partial_{X} L_{k}-\frac{d}{d s}\left(\partial_{\dot{X}} L_{k}\right)=0,
$$

which means

$$
\begin{gathered}
{\left[\partial_{X} L_{k}-\frac{d}{d s}\left(\partial_{\dot{X}} L_{k}\right)\right]_{|r-k|}=0,} \\
{\left[\partial_{X} L_{k}-\frac{d}{d s}\left(\partial_{\dot{X}} L_{k}\right)\right]_{|r-k|+2}=0,} \\
\vdots \\
{\left[\partial_{X} L_{k}-\frac{d}{d s}\left(\partial_{\dot{X}} L_{k}\right)\right]_{r+k}=0 .}
\end{gathered}
$$

Observe that if $k=0$, eq. (28) implies $\left(\begin{array}{l}n \\ k\end{array}\right)=\left(\begin{array}{l}n \\ 0\end{array}\right)=1$ equation, whereas the variation of $A_{r}$ implies $\left(\begin{array}{l}n \\ r\end{array}\right)$ arbitrary variations. Then $\left(\begin{array}{l}n \\ r\end{array}\right) \times 1=\left(\begin{array}{l}n \\ r\end{array}\right)$ and we conclude the existence of $\left(\begin{array}{l}n \\ r\end{array}\right)$ Euler-Lagrange equations, namely, one for each of the $\left(\begin{array}{l}n \\ r\end{array}\right)$ components of $\left[\partial_{X_{r}} L_{0}-\right.$ $\left.\frac{d}{d s}\left(\partial_{\dot{X}_{r}} L_{0}\right)\right] \in \sec \wedge^{r}(T M)$. The same happens with $k \neq 0$ and $r=0$ since in this case we have $\left(\begin{array}{l}n \\ k\end{array}\right) \times 1=\left(\begin{array}{l}n \\ k\end{array}\right)$ Euler-Lagrange equations for $\left[\partial_{X_{r}} L_{k}-\frac{d}{d s}\left(\partial_{\dot{X}_{r}} L_{k}\right)\right] \in \wedge^{k}(T M)$ which has $\left(\begin{array}{l}n \\ k\end{array}\right)$ components. 
We now must extend the above reasoning for $k \neq 0, r \neq 0$. Observe that in this general case we need

$$
\begin{aligned}
\sum_{\ell=0}^{\frac{1}{2}[r+k-|r-k|]}\left(\begin{array}{c}
n \\
|r-k|+2 \ell
\end{array}\right) & =\left(\begin{array}{c}
n \\
|r-k|
\end{array}\right)+\left(\begin{array}{c}
n \\
|r-k|+2
\end{array}\right)+\ldots+\left(\begin{array}{c}
n \\
r+k
\end{array}\right) \\
& \leq\left(\begin{array}{l}
n \\
r
\end{array}\right)\left(\begin{array}{l}
n \\
k
\end{array}\right) ; \quad n, k \leq n, r+k \leq n
\end{aligned}
$$

in order to deduce from eq. (27) the validity of eq. (28). This happens because eq. (28) is equivalent to eq. (29) which is a set of $\sum_{\ell=0}^{\frac{1}{2}[r+k-|r-k|]}$ Euler-Lagrange like equations, and from eq. (27) we can deduce only $\left(\begin{array}{l}n \\ r\end{array}\right)\left(\begin{array}{l}n \\ k\end{array}\right)$ equations of the Euler-Lagrange type.

Now, eq. (30) has been tested in a computer program to be true, and we conclude for the validity of eq. (28), the multivector Euler-Lagrange equation. Indeed, eq. (28) is valid also if $X$ is a general multivector field over $\sigma$, and we conclude that the principle of stationary action with $L=\sum_{k} L_{k}$ produces the general multivector Euler-Lagrange equation

$$
\partial_{X} L-\frac{d}{d s}\left(\partial_{\dot{X}} L\right)=0 .
$$

6.1 Multivector Lagrangian formulation of the superparticle equations of motion. Here $\left\{e_{\mu}\right\}, \mu=0,1,2,3$ is a comoving frame for $\sigma$ in Minkowski spacetime. Consider the bivector valued Lagrangian

$$
L_{S}=\frac{1}{2} e_{\mu} \wedge \dot{e}_{\mu}-\frac{1}{2} \omega_{\mu \nu} e_{\mu} \wedge e_{\nu},
$$

where the $\omega_{\mu \nu}$ are funcions over $\sigma$. From eq. (31) we have four multivector Euler-Lagrange equations $\partial_{e_{\mu}} L_{S}-(d / d s)\left(\partial_{\dot{e}_{\mu}} L_{S}\right)=0$.

Taking into acount that if $x$ and $b$, are vectors then $\partial_{x}(x \wedge b)=3 b$ and $\partial_{x}(x \cdot a)=0$ an easy calculation gives $e_{\mu}=-\omega_{\mu \nu} e^{\nu}$. Defining $\Omega=\frac{1}{2} \omega_{\mu \nu} e^{\mu} \wedge e^{\nu}$ we arrive at

$$
\dot{e}_{\mu}=\Omega \cdot e_{\mu},
$$

which we recognize as the superparticle equations of motion (eq. (6)). For particular values of $\omega_{\mu \nu}$, eq. (33) can be identified with Frenet equations.

6.2 Barut and Zanghi Lagrangian. Let $\psi$ be a Dirac-Hestenes spinor field over $\sigma^{8}$ and $A \in \sec \wedge^{1}(T M) \subset \sec \mathcal{C} \ell(M, g)$ the electromagnetic potential. The following Lagrangian defines the Barut-Zanghi model [21]

$$
L_{B Z}=\left\langle\tilde{\psi} \dot{\psi} \gamma_{2} \gamma_{1}+p\left(\dot{x}-\psi \gamma_{0} \tilde{\psi}\right)+e A \psi \gamma_{0} \tilde{\psi}\right\rangle_{0},
$$

where $x=x^{\mu}(\tau) \gamma_{\mu}$ is the parametric equation for $\sigma, \tau$ being proper time in the "center of mass". Then, the multivector Euler-Lagrange equation (eq. (31)) gives

$$
\dot{\psi} \gamma_{1} \gamma_{2}+\Pi \psi \gamma_{0}=0, \quad \dot{x}=\psi \gamma_{0} \tilde{\psi}, \quad \dot{\Pi}=e F \cdot \dot{x},
$$

where $\Pi=p-e A$ is the kinetic momentum.

\footnotetext{
${ }^{8}$ In the Barut-Zanghi model $\psi$ can be a singular spinor also. See [29].
} 
7. Frenet equations and the Berezin-Marinov model in four dimensional spacetime. Here we show the connection of the Clifford bundle formalism and the concept of multivector derivatives with the Berezin supercalculus. (See also $[17,30]$ )

In 1977 Berezin [3] introduced the following calculus now known as supercalculus. Let $\xi_{i}, i=1, \ldots, n$ be the generations of the Grassmann algebra $\mathcal{G}_{n}$. Then, $\left\{\xi_{i}, \xi_{j}\right\}=0$, where $\{$,$\} is the anticommutator.$

Obviously, we have the following isomorphism

$$
\xi_{i} \leftrightarrow e_{i} ; \xi_{i} \xi_{j} \leftrightarrow e_{i} \wedge e_{j},
$$

where $e_{i}$ is are orthonormal vector generations of the Clifford algebra $\mathcal{C} \ell_{p, q}, p+q=n$. (We leave $p, q$ unespecified at this moment). With the identification above whatever combination of Grassmann variables is isomorphic to a certain multivector.

Berezin introduced the differentiation by the rules

$$
\frac{\vec{\partial} \xi_{j}}{\partial \xi_{i}}=\delta_{i j} ; \xi_{j} \frac{\overleftarrow{\partial}}{\partial \xi_{i}}=\delta_{i j}
$$

Introducing the reciprocal basis $\left\{e^{i}\right\}, e^{i} \cdot e_{j}=\delta_{j}^{i}$ we have

$$
\begin{gathered}
\left.\frac{\vec{\partial}}{\partial \xi_{i}} \leftrightarrow e^{i}\right\lrcorner=e^{i} \cdot, \\
\frac{\overleftarrow{\partial}}{\partial \xi_{j}} \leftrightarrow\left\llcorner e^{j}=\cdot e^{j},\right.
\end{gathered}
$$

where $\lrcorner$ is the left contraction and $L$ the right contraction (see $[8,21]$ ).

We can immediately verify with the identification given by eq. (37) and eq. (38) that differentiation in the Berezin calculus satisfies the so called graded Leibniz rule. Now if $f(\xi)=f\left(\xi_{1}, \ldots, \xi_{n}\right)$ is a general Grassmann function

$$
f(\xi)=f_{0}+f_{i} \xi^{i}+\frac{1}{2} f_{i j} \xi^{i} \xi^{j}+\ldots \frac{1}{n !} f_{i_{n} \ldots i_{n}} \xi^{i_{n}} \ldots \xi^{i_{n}}
$$

Berezin defined integration by the rules

$$
\int 1 d \xi_{i}=0, \quad \int \xi_{i} d \xi_{i}=1, \forall i
$$

$$
\int f\left(\xi_{1} \ldots \xi_{n}\right) d \xi_{n} d \xi_{n-1} \ldots d \xi_{1}=f(\xi) \frac{\overleftarrow{\partial}}{\partial \xi_{n}} \frac{\overleftarrow{\partial}}{\partial \xi_{n-1}} \ldots \frac{\overleftarrow{\partial}}{\partial \xi_{1}}
$$

$f(\xi)$ is clearly isomorphic to a multivector $F$ with the same coeficients as in eq. (39) and eq. (40) is equivalent to

$$
\left(\ldots \left(\left(F \llcorner e ^ { n } ) \llcorner e ^ { n - 1 } ) \llcorner \ldots ) \left\llcornere^{1}=F\left\llcorner E^{n},\right.\right.\right.\right.\right.
$$

where $E^{n}=e^{n} \wedge e^{n-1} \ldots \wedge e^{1}$. With this identification all supercalculus as presented, e.g., in DeWitt [41] reduces to elementary algebraic identities for multivector functions, i.e., for sections of the Clifford bundle. This also shows that superfields, first introduced by Salam and Strathdee [35] are isomorphic to sections of the Clifford bundle. Indeed to 
the superfield

$$
A(x, \xi)=A_{0}(x)+\left(A_{1}(x)\right)_{i} \xi^{i}+\left(\frac{1}{2} A_{2}(x)\right)_{i j} \xi^{i} \xi^{j}+\ldots+\left(\frac{1}{n !} A_{n}(x)\right)_{\mu_{1} \ldots \mu_{n}} \xi^{\mu_{1}} \ldots \xi^{\mu_{n}}
$$

it corresponds $\mathcal{C}(x) \subset \sec \mathcal{C} \ell(M, g)$ given by

$$
\mathcal{C}(x)=A_{0}(x)+\left(A_{1}(x)\right)_{i} e^{i}+\left(\frac{1}{2} A_{2}(x)\right)_{i j} e^{i} e^{j}+\ldots+\left(\frac{1}{n !} A_{n}^{(x)}(x)\right)_{\mu_{1} \ldots \mu_{n}} e^{\mu_{1}} \ldots e^{\mu_{n}}
$$

and we arrive at the conclusion that a superfield has already been discovered since as we already know the Dirac-Hestenes spinor field can be represented in a given basis by an even section of the Clifford bundle. These topics are discussed in details in [33]. We also call the reader's attention that the generalized electromagnetic field of a theory of magnetic monopoles $[23,24]$ is also a superfield. To end this section we write a BerezinMarinov's like Lagrangian [3] for a spinning particle in Minkowski spacetime as

$$
L_{B M}=\frac{1}{2} \dot{\xi}^{\mu} \xi_{\mu}-\frac{1}{2} \omega_{\mu \nu} \xi^{\mu} \xi^{\nu},
$$

where $\xi_{\mu}, \mu=0,1,2,3$ are Grassmann fields over $\sigma$, and $\omega_{\mu \nu}$ are functions over $\sigma$, which in the original Berezin-Marinov model are constant functions.

With the isomorphism defined by eq. (36), namely $\xi_{\mu} \mapsto e_{\mu}$ where $\left\{e_{\mu}\right\}$ is an orthonormal frame over $\sigma$ we get the isomorphism

$$
L_{B M} \simeq L_{S},
$$

where $L_{S}$ is the bivector Lagrangian defined by eq. (74).

From the identification $L_{B M} \mapsto L_{S}$ it becomes clear that in Berezin-Marinov Lagrangian in four dimensional Minkowski spacetime can produce a Dirac-Hestenes equation as we discussed in Section 3. (Compare this with the original Berezin-Marinov model, where it is necessary to use a pentadimensional Grassmann algebra in order to obtain the Dirac equation after quantization).

We think that these nice results are important because they shed a new light on the very abstract formalism of superfields.

We call the reader's attention that in $[17,30]$ it is developed the Lagrangian formalism for Clifford fields and also a study of the Dirac-Hestenes equation in a Riemann-Cartan spacetime [30].

8. Multivector Hamiltonian formalism. The multivector Hamiltonian formalism has been originally introduced by Hestenes [16]. Here we present our version of the theory together with an explicit example, i.e., we present the multivector Hamiltonian formulation of the Barut-Zanghi model [1].

For a multivector Lagrangian $L_{k}=L_{k}(X, \dot{X})$, with $X=X_{r} \in \sec \wedge^{r}(T M) \subset$ $\sec \mathcal{C} \ell(M, g)$ the multivector Euler-Lagrangian equations (eq. (70)) contains equations with grades $|r-k|,|r-k|+2, \ldots, r+k$. Usually, to pass from the Lagrangian to the Hamiltonian formalism we define

$$
P=\partial_{\dot{X}} L
$$


as the canonical moment with $P=P(X, \dot{X})$ and then we eliminate $\dot{X}(X, P)$. However in the case where $L=L_{k}$ we have

$$
\partial_{\dot{X}} L_{k}=\left\langle\partial_{\dot{X}} L_{k}\right\rangle_{|r-k|}+\left\langle\partial_{\dot{X}} L_{k}\right\rangle_{|r-k|+2}+\ldots+\left\langle\partial_{\dot{X}} L_{k}\right\rangle_{r+k},
$$

or

$$
P=\langle P\rangle_{|r-k|}+\langle P\rangle_{|r-k|+2}+\ldots+\langle P\rangle_{r+k} .
$$

Since $\left.\dot{X} \in \sec \wedge^{r}(T M) \subset \sec \mathcal{C} \ell(M, g)\right)$ and $P$ is a sum of inhomogeneous multivectors, it is clear that we cannot in general invert $P=P(X, \dot{X})$ and write $\dot{X}=\dot{X}(X, P)$. When this can be done we can have a multivector Hamiltonian. A nice example is discussed in detail in [17] where other applications of Clifford algebras to supersymmetry are also discussed. For what follows we restrict the Hamiltonian formalism to the case of scalar valued Lagrangians,i.e., $L(X, \dot{X})=\langle L(X, \dot{X})\rangle_{0}$, with $X$ a multivector field over $\sigma$. Without loss of generality we take $X=\langle X\rangle_{r}$. In this case $P=\partial_{\dot{X}} L$ satisfies $P=\langle P\rangle_{k}$. From $P=P(X, \dot{X})$ we suppose that we can invert the equation and obtain $\dot{X}=\dot{X}(X, P)$. Then, we define the Hamiltonian by

$$
H=\left(\dot{X} * \partial_{\dot{X}} L-L\right)_{\mid \dot{X}=X(X, P)} .
$$

It is clear that $H=\langle H\rangle_{0}$ and we write eq. (41) as

$$
H=\dot{X} * P-L=P * \dot{X}-L .
$$

Now, calculating $\partial_{X} H$ we obtain

$$
\begin{aligned}
\eta * \partial_{X} H & =\eta * \partial_{X}(\dot{X} * P-L)=\left(\eta * \partial_{X} \dot{X}\right) * L-\left(\eta * \partial_{X}\right) L \\
& =-\eta * \frac{d}{d s}\left(\partial_{\dot{X}} L(X, \dot{X})\right)=-\eta * \frac{d}{d s} P=-\eta * \dot{P} .
\end{aligned}
$$

Then,

$$
\dot{P}=-\partial_{X} H
$$

Now, calculating $\partial_{P} H$ we get,

$$
\eta * \partial_{P} H=\eta * \partial_{P}[\dot{X}(X, P) * P-L(X, \dot{X}(X, P)]=\eta * \dot{X},
$$

and then

$$
\dot{X}=\partial_{P} H
$$

We then have Hamiltonian's equation for the multivectors, $X, P$.

We can even produce a symplectic structure with the above formalism as done originally by Hestenes [16]. In what follows we prefer to present the theory by giving the multivector Hamiltonian formulation of the Barut-Zanghi model.

9. Multivector Hamiltonian formulation of Barut-Zanghi model ${ }^{9}$. Th BarutZanghi model defined by the Lagrangian given by eq. (34), i.e.,

$$
L=\left\langle\tilde{\psi} \dot{\psi} \gamma_{2} \gamma_{1}+p\left(\dot{x}-\psi \gamma_{0} \tilde{\psi}\right)+e A \psi \gamma_{0} \tilde{\psi}\right\rangle_{0}
$$

\footnotetext{
${ }^{9}$ A preliminary announcement of this section appears in [28].
} 
From eq. (44) we can write

$$
\left\langle(p-e A) \psi \gamma_{0} \tilde{\psi}\right\rangle_{0}=\langle\bar{\psi} \dot{\psi}\rangle_{0}-\langle p \dot{x}\rangle_{0}-L,
$$

where we defined $\bar{\psi}=\gamma_{2} \gamma_{1} \tilde{\psi}$.

$\mathrm{Eq}(45)$ looks like a Legendre transformation; we have $p$ and $\bar{\psi}$ as the momentum canonically conjugate to $x$ and $\psi$, respectively, and the Hamiltonian $H=H(x, p, \psi, \tilde{\psi})$ is

$$
H=\left\langle(p-e A) \psi \gamma_{0} \tilde{\psi}\right\rangle_{0}=\left\langle(p-e A) \psi \gamma_{0} \gamma_{1} \gamma_{2} \tilde{\psi}\right\rangle_{0}
$$

Hamiltonian equations are:

$$
\begin{gathered}
\dot{x}=\partial_{p} H, \quad \dot{p}=-\partial_{x} H, \\
\dot{\psi}=\partial_{\bar{\psi}} H, \quad \dot{\bar{\psi}}=-\partial_{\psi} H,
\end{gathered}
$$

It is trivial to verify that eq. (47) and eq. (48) with the Hamiltonian (46) give of BZ equations, i.e., eq. (35).

Let us show how to give a symplectic structure to the phase space of $B Z$ model illustrating therefore the general method given by Hestenes [16]. First, note that an equation like $\dot{x}=\partial_{p} H$ implies $\gamma^{\mu} \dot{x}_{\mu} H$, or $\dot{x}_{\mu}=\partial_{p^{\mu}} H$. Now take a basis $\left\{E_{0}, E_{1}, E_{2}, E_{3}\right\}$ of $\mathbb{R}^{4}$ such that $E_{a} \cdot E_{b}=\delta_{a b}(a=0, \ldots, 3)$ and define $X=\sum_{a} x_{a} E_{a}$; take another copy of $\mathbb{R}^{4}$ and a basis $\left\{E_{0}^{\prime}, E_{1}^{\prime}, E_{2}^{\prime}, E_{3}^{\prime}\right\}$ with $E_{a}^{\prime} \cdot E_{b}^{\prime}=\delta_{a b}$ and define $P^{\prime}=\sum_{a} p_{a} E_{a}^{\prime}$. Finally, take $\mathbb{R}^{4} \oplus \mathbb{R}^{4}$ with a basis $\left\{E_{0}, \ldots, E_{3} ; E_{0}^{\prime}, \ldots, E_{3}^{\prime}\right\}$ such that $E_{a} \cdot E_{b}^{\prime}=0(\forall a, b)$. We can give a symplectic structure to $\mathbb{R}^{4} \oplus \mathbb{R}^{4}$ by defining the symplectic bivector $J$

$$
J=\sum_{a} J_{a}=\sum_{a} E_{a} \wedge E_{a}^{\prime} .
$$

Note that $E_{a}^{\prime}=E_{a} \cdot J=-J \cdot E_{a}$ and $E_{a}=-E_{a}^{\prime} \cdot J=J \cdot E_{a}^{\prime}$. Then $X^{\prime}=X \cdot J=$ $-J \cdot X, P=J \cdot P^{\prime}=-P^{\prime} \cdot J$, and we can define

$$
Q=X^{\prime}+P=X \cdot J+P, \quad \partial_{Q}=\partial_{X^{\prime}}+\partial_{P},
$$

from which we can write Hamilton equations (47) as

$$
\dot{Q}=\partial_{Q}^{\prime} H
$$

where $\partial_{Q}^{\prime}=-J \cdot \partial_{Q}=\partial_{P}^{\prime}-\partial_{X}$.

In order to do the same with eq. (48) remember that $\mathbb{R}_{1,3}^{+} \ni \psi=\langle\psi\rangle_{0}+\langle\psi\rangle_{2}+\langle\psi\rangle_{4}$; an equation like $\dot{\psi}=\partial_{\tilde{\psi}} H$ gives $\langle\dot{\psi}\rangle_{0}=\partial_{\langle\tilde{\psi}\rangle_{0}} H,\langle\dot{\psi}\rangle_{2}=\partial_{\langle\dot{\psi}\rangle_{2}} H$ and $\langle\dot{\psi}\rangle_{4}=\partial_{\langle\tilde{\psi}\rangle_{4}}$ - where the second one gives $\left(\langle\dot{\psi}\rangle_{2}\right)_{\mu \nu}=\partial_{\langle\tilde{\psi}\rangle_{2}^{\mu \nu}} H$. Now, take a basis $\left\{F_{0}, F_{1}, \ldots, F_{7}\right\}$ of $\mathbb{R}^{8}$ such that $F_{m} \cdot F_{n}=\delta_{m n}(m, n=0,1, \ldots, 7)$ and define

$$
\Psi=\langle\psi\rangle_{0} F_{0}+\left(\langle\psi\rangle_{2}\right)_{01} F_{1}+\ldots+\left(\langle\psi\rangle_{2}\right)_{23} F_{6}+\langle\psi\rangle_{4} F_{7} ;
$$

take another copy of $\mathbb{R}^{8}$ with a basis $\left\{F_{0}^{\prime}, F_{1}^{\prime}, \ldots, F_{7}^{\prime}\right\}$ such that $F_{m}^{\prime} \cdot F_{n}^{\prime}=\delta_{m n}$ and define

$$
\bar{\Psi}^{\prime}=\langle\bar{\psi}\rangle_{0} F_{0}^{\prime}+\left(\langle\bar{\psi}\rangle_{2}\right)_{01} F_{1}^{\prime}+\ldots+\left(\langle\bar{\psi}\rangle_{2}\right)_{23} F_{6}^{\prime}+\langle\bar{\psi}\rangle_{4} F_{7}^{\prime} .
$$

Take $\mathbb{R}^{8} \oplus \mathbb{R}^{8}$ with a basis $\left\{F_{0}, \ldots, F_{7} ; F_{0}^{\prime}, \ldots, F_{7}^{\prime}\right\}$ such that $F_{m} \cdot F_{n}^{\prime}=0(\forall m, n)$, and define the symplectic bivector $K$ :

$$
K=\sum_{m} K_{m}=\sum_{m} F_{m} \wedge F_{m}^{\prime}
$$


with $F_{m}^{\prime}=F_{m} \cdot K$, etc., just like the previous case. If we define

$$
\Phi=\Psi^{\prime}+\bar{\Psi}=\Psi \cdot K+\bar{\psi}, \quad \partial_{\Phi}=\partial_{\Psi^{\prime}}+\partial_{\bar{\Psi}},
$$

then Hamilton equations (48) can be written as

$$
\dot{\Phi}=\partial_{\Phi}^{\prime} H
$$

where $\partial_{\Phi}^{\prime}=-K \cdot \partial_{\Phi}=\partial_{\tilde{\Psi}}^{\prime}-\partial_{\psi}$.

The final step is to take the space $\left(\mathbb{R}^{4} \oplus \mathbb{R}^{8}\right) \oplus\left(\mathbb{R}^{4} \oplus \mathbb{R}^{8}\right)$ with a basis $\left\{E_{0}, \ldots, E_{3}\right.$; $\left.F_{0}, \ldots, F_{7} ; E_{0}^{\prime}, \ldots, E_{3}^{\prime} ; F_{0}^{\prime}, \ldots, F_{7}^{\prime}\right\}$ with $E_{a} \cdot F_{m}=E_{a} \cdot F_{m}^{\prime}=E_{a}^{\prime} \cdot F_{m}=E_{a}^{\prime} \cdot F_{m}^{\prime} \Phi=$ $\Psi^{\prime}+\bar{\Psi}=\Psi \cdot K+\bar{\psi}=0(\forall a, m)$. The symplectic structure is given by the symplectic bivector $\mathcal{J}$ :

$$
\mathcal{J}=J+K=\sum_{a} E_{a} \wedge E_{a}^{\prime}+\sum_{m} F_{m} \wedge F_{m}^{\prime}
$$

After defining

$$
\Pi=Q+\Phi,
$$

we write Hamilton equations (49) and (50) as

$$
\dot{\Pi}=\partial_{\Pi}^{\prime} H
$$

where $\partial_{\Pi}^{\prime}=\partial_{Q}^{\prime}+\partial_{\Phi}^{\prime}$.

We observe that the Poisson brackets are given by

$$
\{F, G\}=\mathcal{J} \cdot\left(\partial_{\Pi} G, \partial_{\Pi} F\right)
$$

in terms of which Hamilton equations (51) can be written as

$$
\dot{\Pi}=\{H, \Pi\} \text {. }
$$

10. Conclusions. In this paper we showed that Frenet equations are the appropriate equations of motion of a classical spinning particle, and from the spinor form of Frenet equations we even obtain the "classical" Dirac-Hestenes equation. We succeded in giving a multivector Lagrangian formalism for Frenet equations and showed that it is isomorphic to a generalization of the 4-dimensional Minkowski spacetime of the Lagrangian of the famous Berezin-Marinov [3] model, thus providing us with a geometrical interpretation of the supercalculus of Berezin [2] (see also DeWitt [41]) and maybe more important than that, we arrive at a geometrical interpretation for the superfields, since we identify the Dirac-Hestenes spinor field with a superfield (see [33])! These results, we think are not only nice, they shed a new light on the abstract formalism of supercalculus revealing their deep geometric contents in terms of objects living in spacetime, i.e, we don't need to introduce abstract spaces as, e.g., the supermanifolds.

Also our formulation leaves clear the relation of Frenet equations with the BarutZanghi model and shows that the helicoidal motion of the electron which appears as one of the possible solutions in this model (zitterbewegung) is not responsable for the electron spin.

Acknowledgments. The authors are grateful to Dr. Q.A.G. de Souza for useful discussions and to FAPESP and CNPq for research grants. Work was also partially supported by the Slovenian Ministry of Science and Technology. 
Appendix: Multivector derivatives. Here $M$ is Minkowski vector space. Let be $x \in \sec \wedge^{1}(T M) \subset \sec \mathcal{C} \ell(M, g)$ and let be

$$
F: \sec \wedge^{1}(M) \rightarrow \sec \wedge^{r}(M), \quad x \mapsto F(x),
$$

with $F(x)=\langle F(x)\rangle_{r}$.

Definition A1. The vector derivative of $F$ in the direction of $a \in \sec \wedge^{1}(T M)$ $\subset \sec \mathcal{C} \ell(M, g)$ is

$$
a \cdot \partial_{x}\langle F(x)\rangle_{r}=\left.\frac{d}{d t}\langle F(x+t a)\rangle_{r}\right|_{t=0}=\left\langle\left.\frac{d}{d t} F(x+t a)\right|_{t=0}\right\rangle_{r}=\left\langle a \cdot \partial_{x} F(x)\right\rangle_{r} .
$$

Then, by definition, $a \cdot \partial_{x}$ preserves the grade, $a \cdot \partial_{x}\langle F(x)\rangle_{r}=\left\langle a \cdot \partial_{x} F(x)\right\rangle_{r}$.

Definition A2. $\partial_{x} F(x)$ is defined by

$$
\left\langle a\left(\partial_{x} F(x)\right)\right\rangle_{r}=\left\langle\left(a \cdot \partial_{x}\right) F(x)\right\rangle_{r} .
$$

Since $a \in \sec \wedge^{1}(T M) \subset \sec \mathcal{C} \ell(M, g)$ we have, where if $A, B$ are multivectors $A * B=$ $\langle A B\rangle_{0}$ :

$$
\left\langle a \partial_{x} F(x)\right\rangle_{r}=a \cdot\left\langle\partial_{x} F(x)\right\rangle_{r+1}+a \wedge\left\langle\partial_{x} F(x)\right\rangle_{r-1} .
$$

DeFinition A3. $\partial_{x} \wedge F(x)$ and $\partial_{x} \cdot F(x)$ are defined by

$$
\begin{gathered}
\partial_{x} \wedge F(x)=\partial_{x} \wedge\langle F(x)\rangle_{r}=\left\langle\partial_{x} F(x)\right\rangle_{r+1}, \\
\partial_{x} \cdot F(x)=\partial_{x} \cdot\langle F(x)\rangle_{r}=\left\langle\partial_{x} F(x)\right\rangle_{r-1} .
\end{gathered}
$$

Then, we have

$$
\partial_{x} F(x)=\partial_{x} \wedge F(x)+\partial_{x} \cdot F(x) .
$$

Now, let $X \in \sec \wedge^{s}(T M) \subset \sec \mathcal{C} \ell(M, g)$ and

$$
F: \sec \wedge^{s}(T M) \rightarrow \sec \wedge^{r}(T M),
$$

that is, $F(X)=\langle F(X)\rangle_{r}$. We have

Definition A4. The multivector derivative of $F(X)$ in the direction of the multivector $A \in \sec \wedge^{s}(M) \subset \sec \mathcal{C} \ell(M, g)$ or $A$-derivative, for short, is

$$
A * \partial_{X}\langle F(X)\rangle_{r}=\frac{d}{d t}\langle F(X+t A)\rangle_{r \mid t=0}=\left\langle\left.\frac{d}{d t} F(X+t A)\right|_{t=0}\right\rangle_{r}=\left\langle A * \partial_{X} F(X)\right\rangle_{r},
$$

where if $A$ and $B$ are multivectors: $A * B=\langle A B\rangle_{0}$. Accordingly $A * \partial_{X}$ preserves the grade: $A * \partial_{X}\langle F(X)\rangle_{r}=\left\langle A * \partial_{X} F(X)\right\rangle_{r}$.

Definition A5. We define $\partial_{X} F(X)$ such that

$$
\left\langle A\left(\partial_{X} F(X)\right)\right\rangle_{r}=\left\langle\left(A * \partial_{X}\right) F(X)\right\rangle_{r} .
$$

Then

$$
\begin{gathered}
\left\langle A \partial_{X} F(X)\right\rangle_{r}=\left\langle A\left\langle\partial_{X} F(X)\right\rangle_{r-s}\right\rangle_{r}+\left\langle A\left\langle\partial_{X} F(X)_{|r-s|+z}\right\rangle_{r}\right. \\
+\ldots+\left\langle A\left\langle\partial_{X} F(X)\right\rangle_{r+k}\right\rangle_{k}, \\
\partial_{X} F(X)=\partial_{\langle X\rangle_{r}}\langle F(X)\rangle_{r} \\
=\left\langle\partial_{X} F(X)\right\rangle_{r-s}+\left\langle\partial_{X} F(X)\right\rangle_{|r-s|+2}+\ldots+\left\langle\partial_{X} F(X)\right\rangle_{r+k},
\end{gathered}
$$

and we denote $\left\langle\partial_{X} F(X)\right\rangle_{r-s}=\partial_{X} \cdot F(X)$ and $\left(\partial_{X} F(X)\right\rangle_{r+k}=\partial_{X} \wedge F(X)$. 


\section{References}

[1] A. O. Barut and N. Zanghi, Classical model of Dirac electron, Phys. Rev. Lett. 52 (1984), 2009-2012.

[2] F. A. Berezin, The Method of Second Quantization, Academic Press, (1966).

[3] — and M. S. Marinov, Particle spin dynamics as the Grassmann variant of classical mechanics, Ann. Physics, 104 (1977), 336-362.

[4] - Introduction to Supersymmetry, D. Reidel Publ. Co., Dordrecht, (1987).

[5] N. N. Bogolubov, A. A. Logunov and I. T. Todorov, Introduction to Axiomatic Quantum Field Theory, W. A. Benjamin Inc., Reading, Massachusetts (1975).

[6] R. Coquereaux, A. Jadczyk and D. Kastler, Differential and integral geometry of Grassmann algebras, Rev. Math. Phys. 3, (1991), 63-99.

[7] C. J. L. Doran, A. N. Lasenby and S. F. Gull, Grassmann mechanics, multivector derivatives and geometric algebra, in Z. Oziewicz, A. Borowiec and B. Jancewicz (eds), Spinors, Twistors and Clifford Algebra, Kluwer Acad. Publ., Dordrecht (1992).

[8] M. A. Faria-Rosa, E. Recami and W. A. Rodrigues Jr, A satisfactory formalism for magnetic monopoles by Clifford algebras, Phys. Lett. B173 (1986) 233-236.

[9] V. L. Figueiredo, E. C. de Oliveira and W. A. Rodrigues Jr, Covariant, algebraic and operator spinors, Int. J. Theor. Phys. 29 (1990), 371-395.

[10] - - - and -, Clifford algebras and the hidden geometric nature of spinors, Algebras, Groups and Geometries 7 (1990), 153-198.

[11] P. G. O. Freund, Supersymmetry, Cambridge University Press, (1986).

[12] G. Fryberger, On generalized electromagnetism and Dirac algebra, Found. Phys. 19 (1989), 125-159.

[13] F. Gursey, Relativistic kinematics of a classical point particle in spinor form, Il Nuovo Cimento 5 (1957), 784-809.

[14] D. Hestenes and G. Sobczyk, Clifford Algebra to Geometrical Calculus, D. Reidel Publ. Co., Dordrecht (1984).

[15] - , The zitterbewegung interpretation of quantum mechanics, Found. Phys. 20 (1990), 1213-1232.

[16] - Hamiltonian mechanics with geometric calculus, in Z. Oziewicz, A. Borowiec and B. Jancewicz (eds.), Spinors, Twistors and Clifford Algebra, Kluwer Acad. Publ., Dordrecht (1992).

[17] A. N. Lasenby, C. J. L. Doran and S. G. Gull, A multivector derivative approach to Lagrangian field theory, Found. Phys. 23 (1993), 1295-1328.

[18] P. Lounesto, Clifford algebras and Hestenes spinors, Found. Phys. 223 (1993), 12031238.

[19] A. Maia Jr, M. A. Faria-Rosa, E. Recami and W. A. Rodrigues Jr, Magnetic monopoles without strings in the Kähler-Clifford algebra bundle, J. Math. Phys. 31 (1990), 502-505.

[20] C. Misner, K. S. Thorne and J. A. Wheeler, Gravitation, W. H. Freeman and Co., San Francisco (1973).

[21] M. Pavšič, E.Recami, W.A. Rodrigues Jr, G. Macarrone, F. Raciti, and G. Salesi, Spin and electron structure, Phys. Lett. B 318 (1993), 481-488.

[22] W. A. Rodrigues Jr and M. A. Faria-Rosa, The meaning of time in relativity, and Einstein's later view of the twin paradox, Found. Phys. 19, (1989), 705-724. 
[23] W. A. Rodrigues Jr, A. Maia Jr, M. A. Faria-Rosa and E. Recami, The classical problem of the charge pole motion, Phys. Lett. B220 (1989), 195-199.

[24] - - - - , and -, Physico mathematical approach to monopoles without strings, Hadronic Journal. 12 (1989), 187-212.

[25] - A comment on generalized electromagnetism and Dirac algebra, Found. Phys. Lett. 3 (1990), 95-99.

[26] — and Q. A. G. de Souza, A Clifford bundle approach to gravitational theory, in J. C. D'Olivo, E. Nahmed-Achar, M. Rosembaum, M. P. Ryan Jr, L. F. Urrutia and F. Zertuche (eds.): Relativity and Gravitation. Classical and Quantum. Proc. of SILARG VII, pp. 220-226, World Scientific Publ. Co., Singapore (1991).

[27] — and -, The Clifford bundle and the nature of the gravitational field, Found. Phys. 23 (1993), 1465-1490.

[28] — and J. Vaz Jr, Zitterbewegung and electron structure, in F. Brackx, R. Delanghe and H. Serras (eds.), Clifford Algebra and Their Applications in Mathematical Physics, pp 397-404, Kluwer Acad. Publ., Dordrecht (1993).

[29] - - - E. Recami, and G. Salesi, About zitterbewegung and electron structure, Phys. Lett. B 318 (1993), 623-628.

[30] -, Q. A. G. de Souza and J. Vaz Jr, Lagrangian formulation in the Clifford bundle of the Dirac-Hestenes equation on Riemann-Cartan manifold, in P. Letelier and W. A. Rodrigues Jr (eds.): Gravitation. The Spacetime Structure. Proc. of SILARG VIII, Aguas de Lindóia, S.P., Brazil (1993), pp 522-531; World Scientific Publ. Co. (1994).

[31] - - - - , and P. Lounesto, Dirac-Hestenes spinor fields, their covariant derivatives and their formulation on Riemann-Cartan manifolds, RT 64/93 IMECC-UNICAMP, subm for publication.

[32] - and J. Vaz Jr, Classical wave equation of the superparticle interacting with an external electromagnetic field, to be submmited for publication (1995).

[33] - Q. A. G. de Souza and J. Vaz Jr, Spinor fields and superfields as equivalence classes of exterior algebra fields, in R. Ablamowicz and P. Lounesto (eds.) Clifford Algebras and Spinor Structures - Crumeyrole Memorial Volume, Kluwer Acad. Publ., Dordrecht (1995).

[34] R. K. Sachs and H. Wu, General Relativity for Mathematicians, Springer-Verlag, New York (1977).

[35] A. Salam and J. Strathdee, Supergauge transformations, Nucl. Phys. B76 (1974), 477-482.

[36] Q. A. G. de Souza and W. A. Rodrigues Jr, The Dirac operator and the structure of Riemann-Cartan-Weyl spaces, in P. Letelier and W. A. Rodrigues Jr (eds.): Gravitation. The Spacetime Structure. Proc. of SILARG VIII, Aguas de Lindóia, S.P., Brazil (1993), pp 177-210; World Scientific Publ. Co. (1994).

[37] J. Takabayashi, Relativistic hydrodynamics of the Dirac matter, Suppl. Progr. Theor. Phys. 4 (1957), 1-80.

[38] J. Vaz Jr, The Barut and Zanghi model, and some generalizations, Phys. Lett. B 344 (1995), 149-164.

[39] - and W. A. Rodrigues Jr, On the equivalence of Maxwell and Dirac eq uations, and quantum mechanics, Int. J. Theor. Phys., 32 (1993), 945-958.

[40] S. Weinberg, Gravitation and Cosmology, John Wiley \& Sons, Inc., New York (1972).

[41] B. de Witt, Supermanifolds, Cambridge University Press, (1984). 